\title{
Proteomics and bioinformatics approaches for the identification of plasma biomarkers to detect Parkinson's disease
}

\author{
WENWEN DONG ${ }^{1 *}$, CHANG QIU $^{1 *}$, DAWEI GONG ${ }^{1}, \mathrm{XU} \mathrm{JIANG}^{2}$, WAN LIU $^{2}$, \\ WEIGUO LIU ${ }^{2}$, LI ZHANG ${ }^{2}$ and WENBIN ZHANG ${ }^{1}$ \\ Departments of ${ }^{1}$ Functional Neurosurgery and ${ }^{2}$ Neurology, Nanjing Brain Hospital Affiliated to \\ Nanjing Medical University, Nanjing, Jiangsu 210029, P.R. China
}

Received November 16, 2018; Accepted June 27, 2019

DOI: $10.3892 /$ etm.2019.7888

\begin{abstract}
The aim of the present study was to screen for biomarkers of Parkinson's disease (PD) using proteomics and bioinformatics approaches. PD patients were divided into three groups: Those without surgery (PD1 group); those who had undergone deep brain stimulation (DBS) surgery without electrode stimulation (PD2 group); and those who had undergone DBS surgery with 1 month of electrode stimulation (PD3 group). The non-Parkinson control group (CK group) was also involved. Quantitative proteomic analysis of human sera was performed through the use of tandem mass tag markers and liquid chromatography-mass spectrometry (LC-MS)-based techniques. For the proteins with quantitative information, a systematic bioinformatics analysis was then performed, including protein annotation, functional classification, functional enrichment and cluster analysis based on functional enrichment. Of the 739 proteins identified, quantitative information was available for 644. With regard to differential expression, 18 upregulated and 21 downregulated proteins were screened in the PD1/CK comparison group; 12 upregulated and 12 downregulated proteins in the PD2/PD1 comparison group; and 16 upregulated and 19 downregulated proteins in the PD3/PD2 comparison group. Coiled-coil domain-containing protein 154 (CCDC154) and tripartite motif-containing protein 3 (TRIM3) were key proteins involved in the molecular mechanisms of PD, participating in intracellular vesicle, ubiquitin protein ligase
\end{abstract}

Correspondence to: Dr Li Zhang, Department of Neurology, Nanjing Brain Hospital Affiliated to Nanjing Medical University, 264 Guangzhou Road, Nanjing, Jiangsu 210029, P.R. China

E-mail: neuro_zhangli@163.com

Dr Wenbin Zhang, Department of Functional Neurosurgery, Nanjing Brain Hospital Affiliated to Nanjing Medical University, 264 Guangzhou Road, Nanjing, Jiangsu 210029, P.R. China

E-mail: wenbinzhang@njmu.edu.cn

${ }^{*}$ Contributed equally

Key words: proteomics, biomarker, Parkinson's disease, functional enrichment analysis, cluster analysis and transition metal ion-binding activities. After DBS surgery, desert hedgehog protein (DHH) was downregulated, whereas neuropilin-2 (NRP2) was upregulated; these participated in the ensheathment of neurons and the semaphorin receptor complex, respectively. The expression level of chloride intracellular channel protein 1 (CLIC1) was increased after 1 month of electrode stimulation following DBS. By combining proteomic approaches and LC-MS methods, significant proteins including CCDC154, TRIM3, DHH, NRP2 and CLIC1 were detected with high specificity and sensitivity. These may be used as novel biomarkers for early diagnosis of PD and the future development of treatments.

\section{Introduction}

Parkinson's disease (PD) is a long-term degenerative disease of the central nervous system that always affects the motor system (1). In 2015, 6.2 million people were affected by PD, and the mortality rate of PD was $1.89 \%$ in the worldwide (2). The most characteristic symptoms of PD were depression, anxiety, as well as sleep and emotional problems (3). The causes of PD mainly involve genetic and environmental factors (4). Different preventative strategies have been proven to protect against the disease, including anti-oxidants, anti-inflammatory drugs and calcium channel blockers (5). Therefore, monitoring and prevention are important for PD, particularly in individuals above the age of 60 years.

Although medical treatment, surgery and physical therapy may provide relief of the associated symptoms, PD itself is thus far incurable (6). For the development of therapeutic interventions, a clear and detailed understanding of the molecular mechanisms underlying the disease is required. After years of research, numerous mechanisms have been revealed, including damage to the protein degradation system, inflammation, mitochondrial dysfunction and oxidative stress, excitotoxicity and enhanced apoptosis (7). However, the exact combination and timing of these molecular events remain to be fully elucidated.

To date, research on the pathogenesis of PD has relied heavily on toxic and transgenic animal models, the engineering of which is derived from rare neurotoxin-induced and single-gene forms of PD in humans (8). However, these hypothesis-driven approaches have major limitations. Proteomics may provide a novel research paradigm for studying the pathogenesis of PD 
and associated biomarkers. The emergence of neuroproteomics, with its sophisticated techniques and unbiased ability to quantify proteins, provides a methodology with which to study the neuronal changes that are associated with neurodegeneration (9). In addition, Kim et al (10) illustrated the potential of neuromics to profile differences in the distribution of thousands of proteins as a function of PD biomarkers. Of note, Monti et al (11) used network analysis to identify the biological processes that were specifically affected in PD and screened four cellular component complexes, namely the proteasome complex, the protein phosphatase 2A complex, the chaperonin CCT complex and complex III of the respiratory chain.

In the present study, proteomics and bioinformatics techniques were combined to screen for potential biomarkers of PD, using plasma samples from affected patients and healthy individuals. The results may provide novel biomarkers for the early detection of this debilitating disease.

\section{Materials and methods}

\section{Experimental methods}

Samples. Plasma samples from a total of 24 individuals were collected at the Affiliated Brain Hospital of Nanjing Medical University (Nanjing, China) between December 2016 and January 2018. Of these subjects, 12 were patients with PD and assigned to one of the following three treatment status groups: Those without surgery (PD1 group; $n=4)$; those who had undergone deep brain stimulation (DBS) surgery without electrode stimulation (PD2 group; $n=4)$; and those who had undergone DBS surgery with 1 month of electrode stimulation (PD3 group; $n=4)$. All patients were eligible for inclusion if they were diagnosed with idiopathic PD on the basis of the Movement Disorders Society criteria (12), with no dementia. The other plasma samples were from 12 healthy individuals, who made up the non-Parkinson control group (CK group). Healthy individuals were recruited by voluntary registration and medical examination. Blood samples from all study participants were collected into heparin tubes and then centrifuged to obtain the plasma samples, which were then stored at $-80^{\circ} \mathrm{C}$ until use. The general characteristics of the individuals (e.g., mean age and gender ratio) were not significantly different between the patient group (age range: 65-79, median age: 72 and gender: 8 males and 4 females) and CK group (age range: 65-71 median age: 69 and gender: 9 males and 3 females). The present study was approved by the ethics committee of Nanjing Brain Hospital (approval no. 2016-KY009). All patients or their family members provided written informed consent for participation in the study.

Protein extraction and digestion. The plasma samples that had been stored at $-80^{\circ} \mathrm{C}$ were first processed with the ProteoExtract High Abundance Protein Removal Kit (Merck) to remove proteins with high abundance ratios. The gravity self-flow purification column provided with the kit contains an affinity resin mixture (IgG antibody ligand) that may efficiently and specifically bind to and remove $>80 \%$ of the serum albumin and $\mathrm{IgG}$ in the sample. The kit was easy to use, with low cross-contamination. Each column adsorbed up to $2 \mathrm{mg}$ of albumin and $0.7 \mathrm{mg}$ IgG. The residual protein content was detected using bicinchoninic acid methods. The protein solution was first reduced with $10 \mathrm{mM}$ dithiothreitol for $30 \mathrm{~min}$ at $56^{\circ} \mathrm{C}$ and then alkylated with
$22 \mathrm{mM}$ iodoacetamide for $45 \mathrm{~min}$ at room temperature in the dark. The protein was precipitated with $20 \%$ trichloroacetic acid for $2 \mathrm{~h}$ and then washed 3 times with acetone. Trypsin was then added first at the ratio of 1:50 (trypsin:protein) for a 12-h incubation at room temperature, and then at the ratio of 1:100 for an additional 4-h digestion.

Tandem mass tag (TMT) labeling. After digestion, the peptides were desalted on a Strata-X C18 SPE column (Phenomenex) and reconstituted according to the manufacturer's protocol. Different isobaric chemical TMTs were dissolved in anhydrous acetonitrile and then centrifuged. The isobaric TMTs were then added to the peptides obtained, and the mixture was incubated for $2 \mathrm{~h}$ at room temperature to allow for peptide labeling. Subsequently, the sample was desalted and dried by vacuum centrifugation.

Liquid chromatography-mass spectrometry (LC-MS) analysis. The peptides were resolved in solvent A ( $0.1 \%$ formic acid, v/v), and then resolved on an EASY-nLC 1000 ultra-high-performance liquid chromatography (UPLC; UltiMate UHPLC Focused; Dionex; Thermo Fisher Scientific, Inc.) system by gradient elution with a mobile phase consisting of solvent A (aqueous solution of $0.1 \%$ formic acid and $2 \%$ acetonitrile) and solvent B (aqueous solution of $0.1 \%$ formic acid and $90 \%$ acetonitrile). The gradient elution comprised an increase from 7-25\% for $26 \mathrm{~min}, 25-40 \%$ for $8 \mathrm{~min}, 40-80 \%$ for $13 \mathrm{~min}$ and $80 \%$ for $3 \mathrm{~min}$, with the flow rate maintained at $400 \mathrm{nl} / \mathrm{min}$.

The peptides were separated on the UPLC system, injected into a nanospray ionization ion source and then analyzed on a Q Exactive Plus mass spectrometer (Q Exactive; Thermo Fisher Scientific, Inc.) with the ion source voltage set to $2.0 \mathrm{kV}$. The peptide precursors and their secondary fragments were detected and analyzed by high-resolution mass spectrometry, with the Orbitrap mass spectrometer (Thermo Electron; Thermo Fisher Scientific, Inc.) scan range set from 350 to $1,800 \mathrm{~m} / \mathrm{z}$ and the scan resolution set at 70,000 . The scan range of the secondary mass spectrometer was fixed at $100 \mathrm{~m} / \mathrm{z}$ and the scan resolution of the Orbitrap mass spectrometer was set at 17,500. The mode for data acquisition was a data-dependent scan program. In order to increase the effective utilization of mass spectrometry, the automatic gain control was set at $5 \times 10^{4}$, the signal threshold was set at 10,000 ions/sec, the maximum injection time was set at $200 \mathrm{msec}$ and the dynamic exclusion time of the tandem mass scan was set at $30 \mathrm{sec}$ to avoid the parent ion.

Database search. The MaxQuant search engine (v.1.5.2.8; https://www.maxquant.org/maxquant/) was used for researching the secondary mass spectral data (13). The database consisted of three parts: The SwissProt human database with 20,130 sequences, the anti-library and common pollution libraries. The anti-library was added to calculate the false discovery rate caused by random matches. Common pollution libraries were added to the database to eliminate the effects of contaminating proteins in the identification results. The effect of enzyme digestion was set as Trypsin/P; the number of missed sites was set at 2 ; the minimum length of the peptide was set to 7 amino acid residues; the maximum number of modified peptides was set to 5; the ion mass error tolerance values for the first parent of the first search and main search were set as 20 and 5 ppm, respectively; and the mass error tolerance of the secondary 
fragment ions was set as $0.02 \mathrm{Da}$. The cysteine alkylation was set as a fixed modification, whereas the oxidation of methionine and acetylation of the N-terminus were variable modifications. The quantitative method was set as TMT-6plex, and the false discovery rates of protein identification and peptide-spectrum match identification were set to $1 \%$.

\section{Bioinformatics methods}

Trend analysis of proteins. In the present study, plasma samples were collected from patients with PD and the non-Parkinson control group included plasma from 12 healthy individuals (CK group). The expression levels of different proteins in the different status conditions were revealed on the basis of trend models. In this process, the multiple comparative test and Bonferroni's correction were used to control the family-wise error rate.

Protein annotation. To gain a thorough understanding of the proteins identified and quantified in the data, their functions and characteristics were investigated using various methods, namely Gene Ontology (GO), protein domain and Kyoto Encyclopedia of Genes and Genomes (KEGG) pathway annotations, and subcellular location classification. GO annotation is a bioinformatic analysis method that links genes and associated proteins and provides statistical information. In proteomic projects, it is always used as a database with information of genes and proteins. Furthermore, it may provide comprehensive information for protein annotation and classification. Protein domain annotation was also performed on the proteins identified in this study, using the program InterProScan based on the protein sequence algorithm and the corresponding InterPro domain database (v7.4). The InterPro domain database (http://www.ebi.ac.uk/interpro/) is a free web database that integrates information of protein family classification, protein domain classification and protein functional site classification. KEGG is able to map currently known protein interaction networks, including pathways and associated complexes, genes and their products in biological complexes and associated information, as well as other integrated information. In the present study, the KEGG pathway database was used to annotate the protein pathways. The submitted proteins were first annotated using the KEGG online service tool KAAS (https://www.genome.jp/tools/kaas/) (14), and the annotated proteins were then matched to the corresponding pathways in the database via KEGG Mapper (https://www. genome.jp/kegg/mapper.html) (14). Furthermore, the proteins in eukaryotic cells are located on various elements within the cell, with the major subcellular locations being the extracellular region, cytoplasm, nucleus, mitochondrion, Golgi apparatus, endoplasmic reticulum, peroxisome, vacuole, cytoskeleton, nucleoplasm, nuclear matrix and ribosome. The binding of proteins to those elements depends on differences in the membrane structure. Therefore, on the basis of this molecular mechanism, the program WoLF PSORT (https://wolfpsort.hgc. $\mathrm{jp} /$ ) was used to predict the subcellular location of the submitted proteins (15).

Enrichment analysis. The GO annotation of proteins is divided into three major categories: Biological process, cellular component and molecular function. The KEGG database was used for pathway enrichment analysis. InterPro (a resource that provides functional analysis of the family classification, domain and
Table I. Statistical information on the differentially expressed proteins $(\mathrm{P}<0.05)$.

\begin{tabular}{lcc}
\hline $\begin{array}{l}\text { Comparison } \\
\text { between }\end{array}$ & $\begin{array}{c}\text { No. of proteins } \\
\text { upregulated }\end{array}$ & $\begin{array}{c}\text { No. of proteins } \\
\text { downregulated }\end{array}$ \\
two groups & $(>1.2$ fold change $)$ & $(<0.83$ fold change $)$
\end{tabular}

\begin{tabular}{llr}
\hline PD1/CK & 18 & 21 \\
PD2/CK & 23 & 25 \\
PD3/CK & 16 & 19 \\
PD2/PD1 & 12 & 12 \\
PD3/PD1 & 10 & 5 \\
PD3/PD2 & 16 & 19 \\
\hline
\end{tabular}

Groups: PD1, PD patients without surgery; PD2, PD patients who had undergone DBS surgery without electrode stimulation; PD3, PD patients who had undergone DBS surgery with 1 month of electrode stimulation; CK, control group without PD. DBS, deep brain stimulation; PD, Parkinson's disease.

specific locus prediction of protein sequences) was used to analyze the enrichment of functional domains of differentially expressed proteins. Fisher's exact test was used to test the differentially expressed proteins, where the threshold of significance was $\mathrm{P}<0.05$.

Cluster analysis based on protein functional enrichment. Cluster analysis based on functional enrichment was used to investigate potential links and differences in specific functions (including GO, KEGG pathways and protein domains). First, the functional classification information and the corresponding P-value was collected and the functional classes that were significantly enriched were then screened out $(\mathrm{P}<0.05)$ in at least one protein cluster. The filtered P-value matrix was subjected to a logarithmic transformation of $-\log 10$, and the transformed data matrix was then subjected to $\mathrm{Z}$ transformation for each functional classification. Finally, the Z-transformed dataset was analyzed by hierarchical clustering (Euclidean distance, average connected clustering). The clustering association was visualized using heatmap. 2 in the R package (v3.6.0).

\section{Results}

Differentially expressed proteins. In the present study, a total of 739 proteins were identified and the quantitative information was available for 644 of them. Differentially expressed proteins were screened on the basis of a threshold fold change of $>1.2$ or $<0.83$. As presented in Table I, a total of 18 upregulated and 21 downregulated proteins were identified as being differentially expressed between the PD1 and CK groups. In the PD3 subjects who received DBS surgery and 1 month of electrode stimulation, 16 upregulated and 19 downregulated proteins compared with the CK group were identified.

In addition, these differentially expressed proteins were clustered into 5 trend modules (Fig. 1). It is worth noting that in cluster 2 and cluster 5 , the protein expression levels improved or even returned to normal after DBS surgery and 1 month of electrode stimulation treatment. Cluster 2 comprised 96 
Table II. Top 3 GO secondary annotation classifications of differentially expressed proteins in PD patients without surgery vs. control group without PD.

\begin{tabular}{lc} 
A, Upregulated proteins & \\
\hline Category/GO terms & No. of proteins \\
\hline Biological process & \\
Biological regulation & 16 \\
Metabolic process & 15 \\
Single-organism process & 14 \\
Cellular component & \\
Extracellular region & 17 \\
Organelle & 14 \\
Cell & 12 \\
Molecular function & \\
Binding & 16 \\
Catalytic activity & 8 \\
Molecular function regulator & 4 \\
\hline
\end{tabular}

B, Downregulated proteins

\begin{tabular}{lc}
\hline Category/GO terms & No. of proteins \\
\hline Biological process & \\
Biological regulation & 18 \\
Single-organism process & 17 \\
Response to stimulus & 15 \\
Cellular component & \\
Cell & 17 \\
Organelle & 17 \\
Extracellular region & 16 \\
Molecular function & \\
Binding & 17 \\
Catalytic activity & 5 \\
Molecular function regulator & 3 \\
\hline
\end{tabular}

GO, gene ontology; PD, Parkinson's disease.

proteins, including immunoglobulin heavy variable 3-64, coiled-coil domain-containing protein 154 (CCDC154), and tripartite motif-containing protein 3 (TRIM3). Similarly, cluster 5 comprised 56 proteins, including desert hedgehog protein (DHH), neuropilin-2 (NRP2) and chloride intracellular channel protein 1 (CLIC1). Compared with those in the CK samples, the expression levels of proteins in cluster 2 and cluster 5 were downregulated in the PD1 group and increased after treatment.

Protein annotation. In order to gain a thorough understanding of the proteins, detailed annotations were performed on the basis of GO, protein domain and KEGG pathway enrichment analyses, as well as subcellular location classification.
Table III. Subcellular location classification of differentially expressed proteins in PD patients without surgery vs. control group without PD.

A, Upregulated proteins

Subcellular location No. of proteins

Extracellular 15

Endoplasmic reticulum

Plasma membrane

1

Nucleus

1

B, Downregulated proteins

\begin{tabular}{lc} 
Subcellular location & No. of proteins \\
\hline Extracellular & 10 \\
Nucleus & 4 \\
Endoplasmic reticulum & 3 \\
Mitochondria & 2 \\
Peroxisome & 1 \\
Cytoplasm & 1 \\
\hline
\end{tabular}

PD, Parkinson's disease.

GO secondary annotation classification. Statistics were performed on the distribution of the quantified proteins in GO secondary annotation. As presented in Table II, the upregulated proteins in the PD1/CK comparison group were mainly classified in the GO terms biological regulation (no. of proteins, 16), extracellular region (no. of proteins, 17) and binding (no. of proteins, 16), whereas the downregulated proteins were classified in the GO terms biological regulation (no. of proteins, 18), cell (no. of proteins, 17) and binding (no. of proteins, 17).

Subcellular location classification. After performing the subcellular structure prediction and classification statistics for the differentially expressed proteins, the top subcellular locations were obtained. Most of the upregulated proteins in the PD1/CK comparison group were mainly located in the extracellular region (no. of proteins, 15), endoplasmic reticulum (no. of proteins, 1) and plasma membrane (no. of proteins, 1), whereas most of the downregulated proteins were located in the extracellular region (no. of proteins, 10), nucleus (no. of proteins, 4) and endoplasmic reticulum (no. of proteins, 3; Table III).

\section{Functional enrichment analysis}

GO functional enrichment analysis. The results of the GO functional enrichment are presented in Fig. 2. The upregulated proteins in the PD1/CK comparison group were mainly enriched in the insulin-like growth factor ternary complex, serine-type endopeptidase inhibitor activity and bioluminescence modules, whereas the downregulated proteins were involved in the endoplasmic reticulum chaperone complex, complement binding and sequestering of calcium ions modules. 
Cluster 1

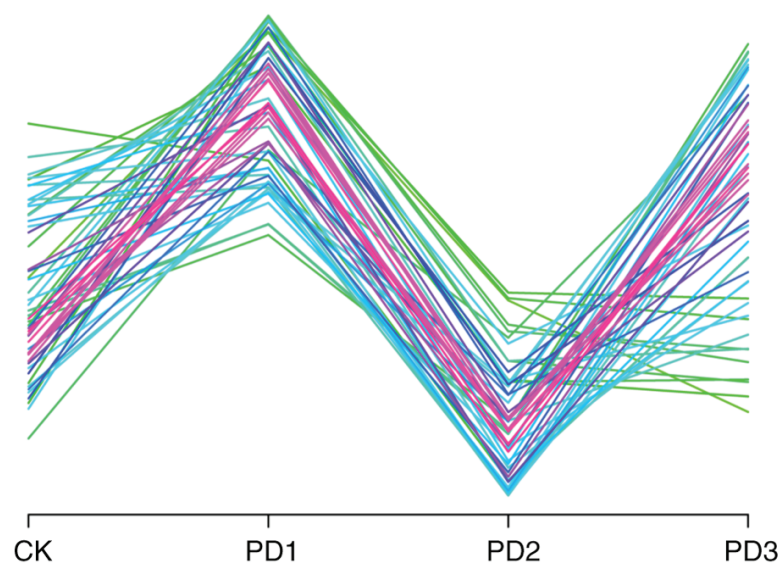

Cluster 3

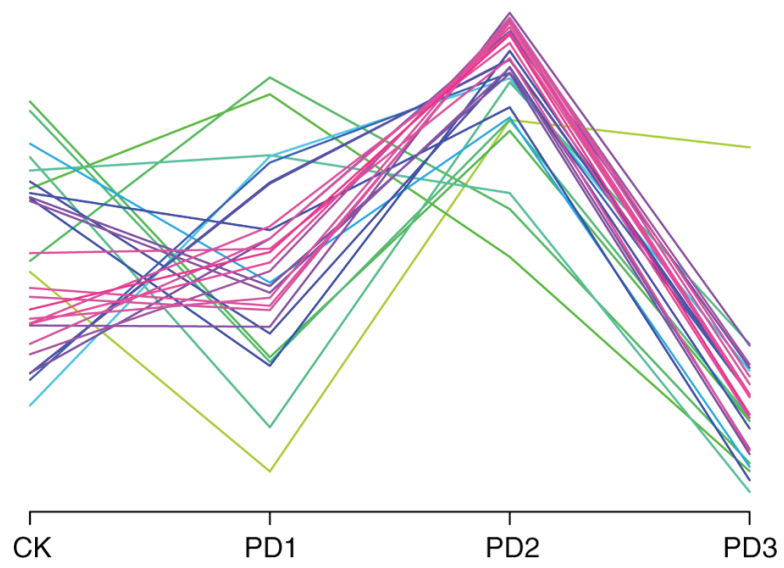

Cluster 5

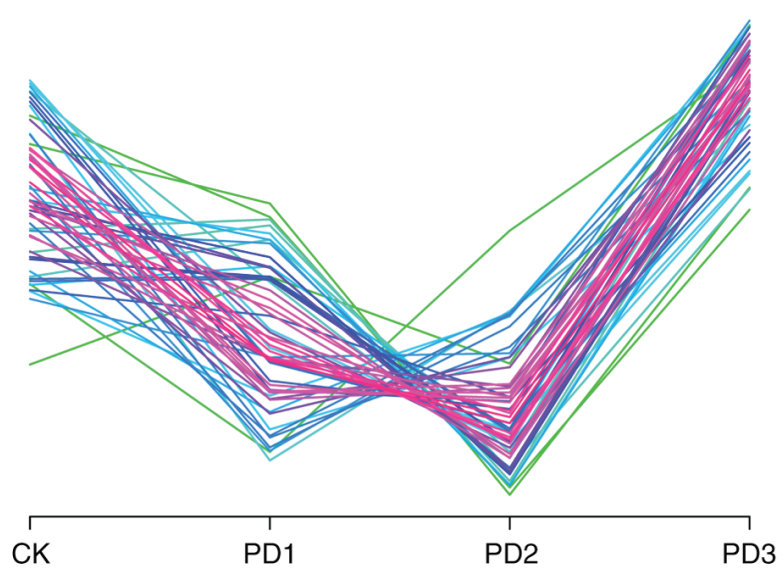

Cluster 2

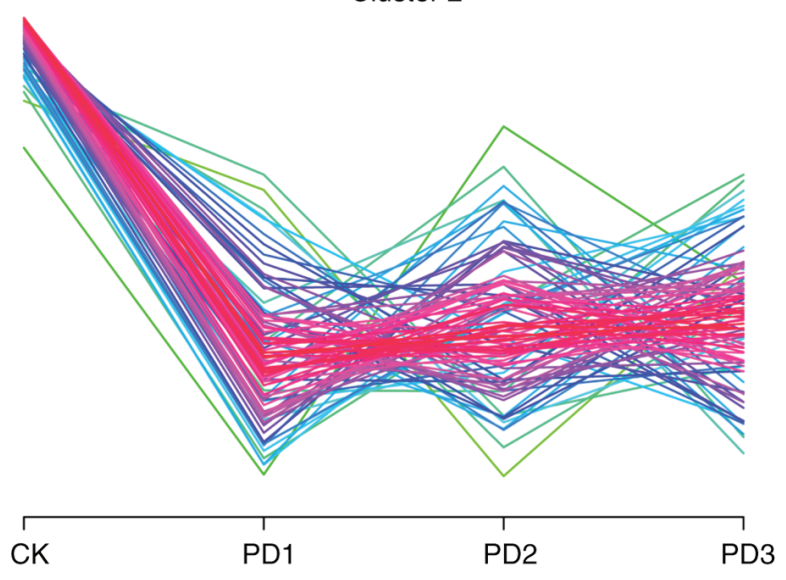

Cluster 4
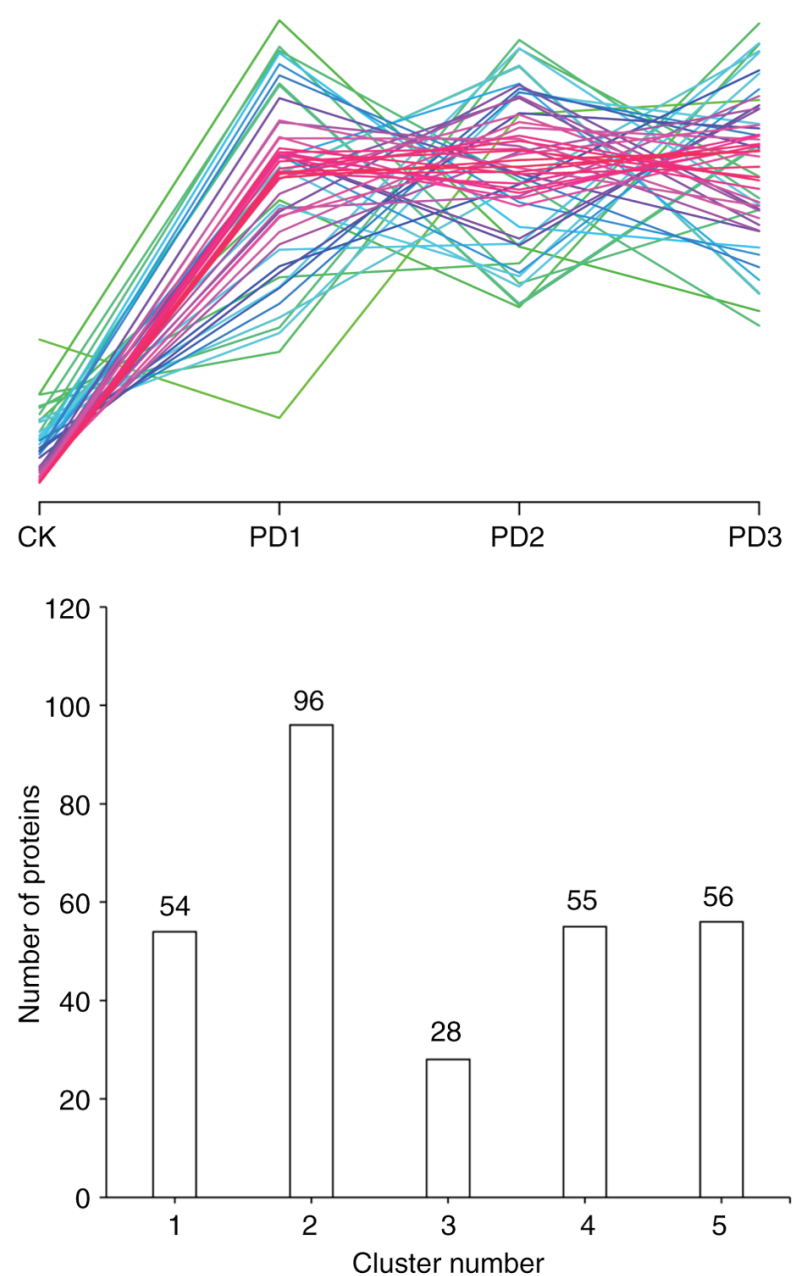

Figure 1. Trend analysis of differentially expressed proteins. The lines represent the expression trend of each protein. The column chart represents the number of proteins in each cluster. Groups: PD1, PD patients without surgery; PD2, PD patients who had undergone DBS surgery without electrode stimulation; PD3, PD patients who had undergone DBS surgery with 1 month of electrode stimulation; CK, control group without PD. DBS, deep brain stimulation; PD, Parkinson's disease.

KEGG pathway enrichment analysis. KEGG pathway enrichment analysis was also performed for the differentially expressed proteins. The upregulated proteins in the PD1/CK comparison group were mapped to proteoglycans in cancer and pathways in cancer, whereas the downregulated proteins were mapped to protein processing in endoplasmic reticulum and systemic lupus erythematous (Fig. 3). Of these, the proteins mapped to proteoglycans in cancer included stromal cell-derived factor 1 , insulin-like growth factor 1 and Indian hedgehog protein. The downregulated proteins mapped to protein processing in 


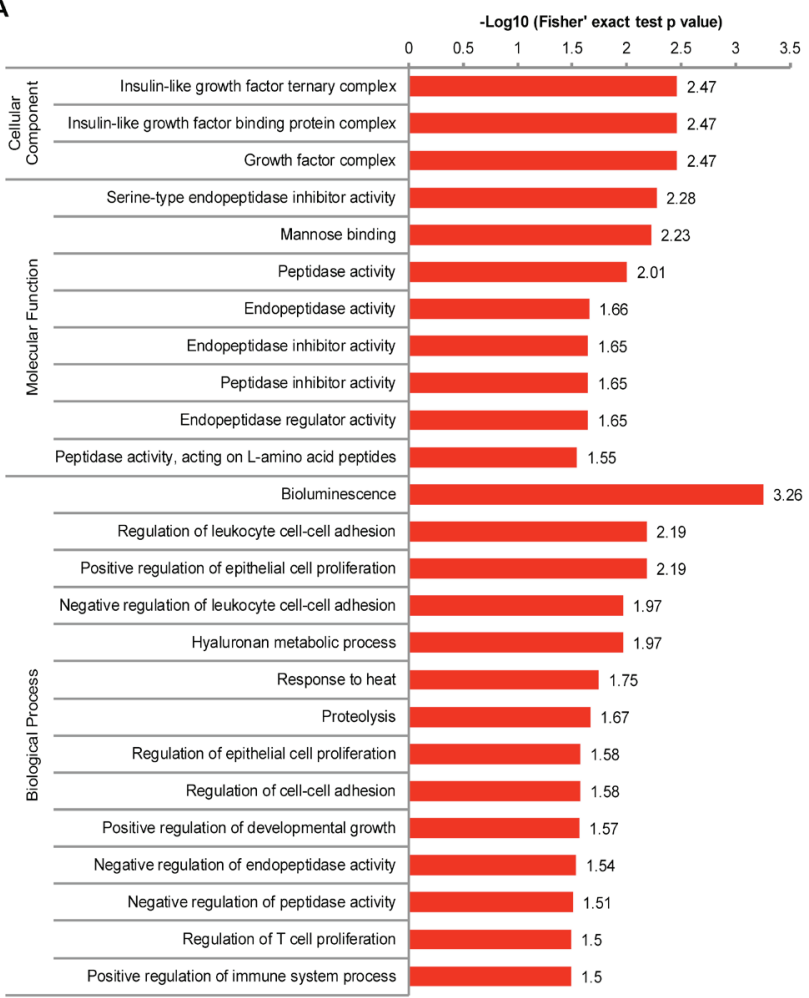

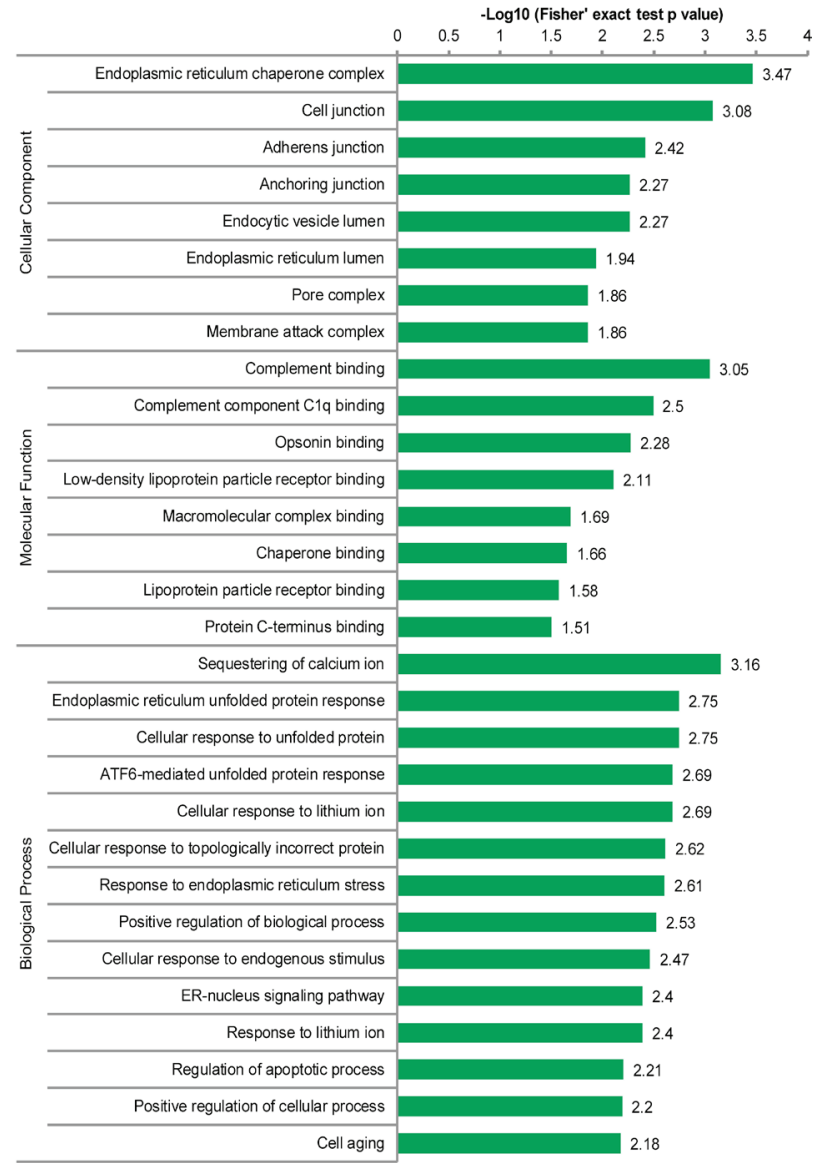

Figure 2. Gene ontology functional enrichment analysis of differentially expressed proteins in PD patients without surgery vs. control group without PD. (A) The red bands represent upregulated proteins. (B) The green bands represent downregulated proteins. PD, Parkinson's disease.

A

$-\lg$ (Fisher's exact test $\mathrm{p}$ value)

hsa05200 pathways in cancer $\quad 1.3$

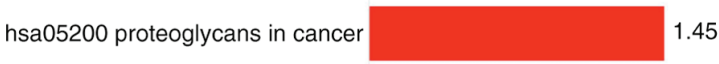

$\begin{array}{lllllll}1.2 & 1.25 & 1.3 & 1.35 & 1.4 & 1.45 & 1.5\end{array}$

B

$-\lg$ (Fisher's exact test $\mathrm{p}$ value)

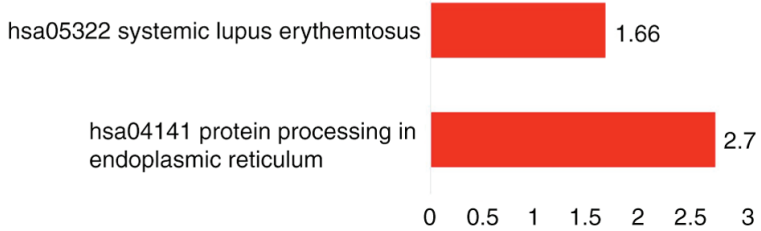

Figure 3. Kyoto Encyclopedia of Genes and Genomes pathway enrichment analysis of differentially expressed proteins in PD patients without surgery vs. control group without PD. (A) Upregulated proteins; (B) Downregulated proteins. PD, Parkinson's disease; hsa, Homo sapiens.

endoplasmic reticulum contained endoplasmin, calreticulin, protein disulfide isomerase and hypoxia-upregulated protein 1 .

Protein domain enrichment analysis. Protein domain refers to the repeated occurrence of certain components in different protein molecules, with similar sequence, structure and function. The length of the domain is usually between 25 and 500 amino acids. In the present study, the upregulated proteins in the PD1/CK comparison group were mainly annotated to the collectin, C-type lectin-like domain, whereas the downregulated proteins were annotated to the Factor $\mathrm{I} / \mathrm{membrane}$ attack complex domain (Fig. 4).

Cluster analysis. On the basis of the P-value obtained from the enrichment analysis, the hierarchical clustering method was used to group together the associated functions in different groups and plot them as a heatmap. The horizontal axis of the heatmap represents the enrichment test results of different groups and the vertical axis represents the description of functions (GO, KEGG pathway, protein domain). The color blocks, corresponding to the differentially expressed proteins and functional descriptions of different groups, indicate the degree of enrichment.

As presented in Figs. 5 and 6, the upregulated proteins in the PD1/CK comparison group were clustered under mannose binding, C-type lectin fold,C-type lectin-like, and cysteine-rich flanking region, $\mathrm{C}$-terminal. The upregulated proteins in the PD3/PD1 comparison group were clustered under purine ribonucleoside triphosphate binding and nucleoside binding. The downregulated proteins in the PD1/CK comparison group were clustered in the factor $\mathrm{I} / \mathrm{membrane}$ attack complex and membrane attack complex/perforin domains. 
A

$-\lg$ (Fisher's exact test $p$ value)

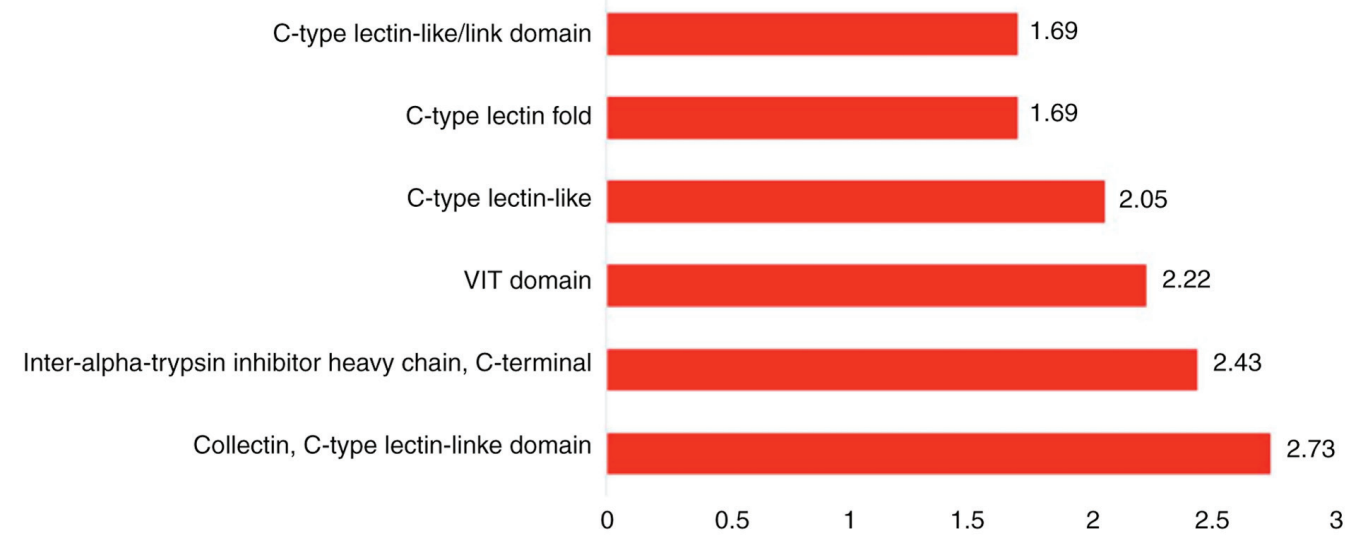

B

$-\lg$ (Fisher's exact test $\mathrm{p}$ value)

Tissue inhibitor of metalloproteinases-like, OB-fold
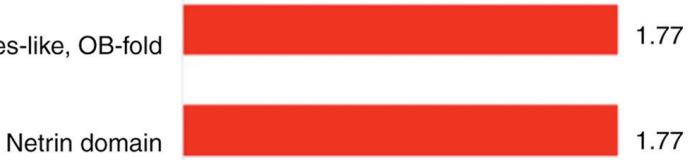

Netrin module, non-TIMP type

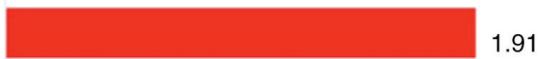

Memberane attact complex component/perforin (MACPF) domain

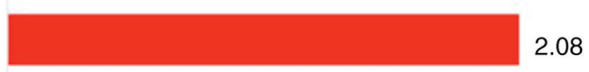

Factor I/memberance attact complex

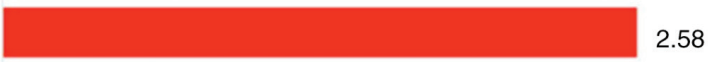

0

0.5

1.5

2

2.5

3

Figure 4. Protein domain enrichment analysis of differentially expressed proteins in PD patients without surgery vs. control group without PD. (A) Upregulated proteins; (B) Downregulated proteins. PD, Parkinson's disease.

\section{Discussion}

It has been indicated that olfactory, sleep, mental and cognitive disorders as well as autonomic dysfunction commonly occur in patients with PD and appear in different periods (16). Although PD does not shorten the life span of affected patients, it has a serious impact on their quality of life and brings heavy burdens to the patients and their family members (17). As the etiology and pathogenesis of PD remain to be fully elucidated, research studies to elucidate these are of utmost importance. PD is currently considered to be the result of a combination of genetic and environmental factors (18). Numerous studies have suggested that degeneration of the dopaminergic neurons of the substantia nigra in patients with PD may be induced by a series of mechanisms, including mitochondrial dysfunction, oxidative stress, excitotoxicity, neurotrophic factor deficiency, immune regulation abnormalities and cell apoptosis (19). Epidemiological studies have demonstrated that $10-15 \%$ of patients with PD had a family history of the disease. At least 13 pathogenic genes associated with PD have been identified by studying the mechanism of genetic factors in PD pathogenesis, indicating that the pathogenesis of the disease is closely linked to genetic factors. Therefore, studies on the occurrence and development of PD at the molecular level are of great significance for its prevention, control and treatment (20). Proteomic profiling is a sophisticated bioinformatics method for data analysis. In the present study, the protein profiles of 12 plasma samples from PD patients at different treatment stages and of 12 samples from healthy controls were analyzed. Compared with their expression in the CK group, CCDC154 and TRIM3 were downregulated in the PD1 samples. After DBS surgery, DHH was downregulated, whereas NRP2 was upregulated. Furthermore, the expression level of CLIC1 was increased after 1 month of electrode stimulation.

CCDC154 is a novel functional protein that has been confirmed to be associated with cell proliferation (21). In the present study, it was enriched in intracellular vesicles, intracellular organelles and cytoplasmic vesicles. Volles and Lansbury (22) confirmed that the pathogenesis of PD may involve membrane permeabilization by protofibrillar alpha-synuclein, and its extent may strongly depend on the in vivo conditions. In the Drosophila model of PD, synaptic vesicle proteomes contributed to the phosphorylation of synaptic vesicle proteins in the brain (23). Furthermore, Volz et al (24) indicated that vesicular monoamine transporter-2 in cytoplasmic and membrane-associated vesicles may affect transport kinetics and respond to pharmacological manipulation in vivo.

TRIM3 is a member of the tripartite motif, which is located on cytoplasmic filaments. In the present study, TRIM3 was downregulated in the PD1 samples and was 
A
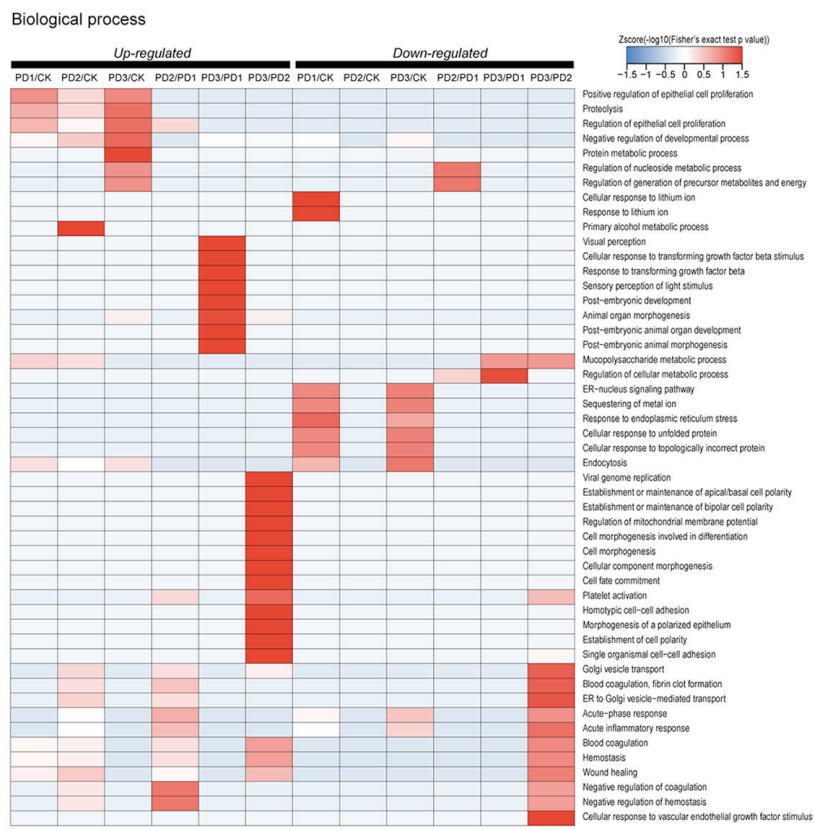

c

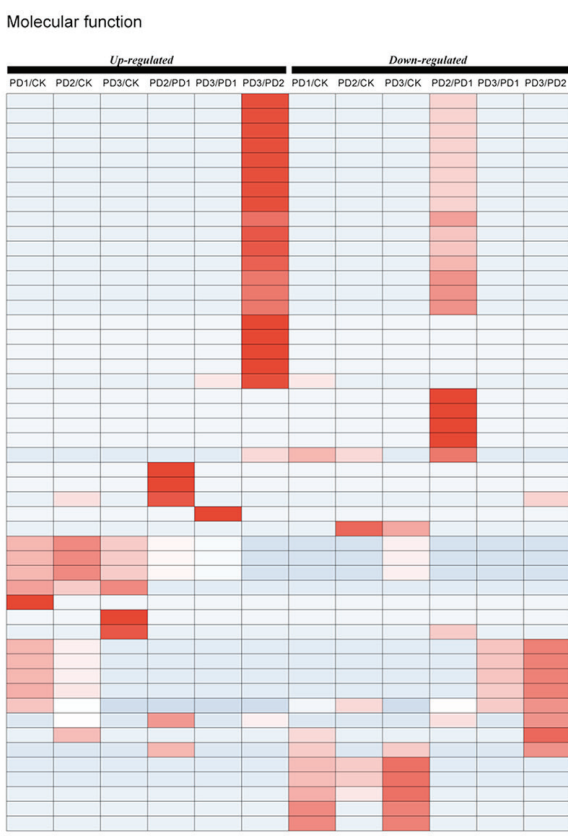
Cellular component

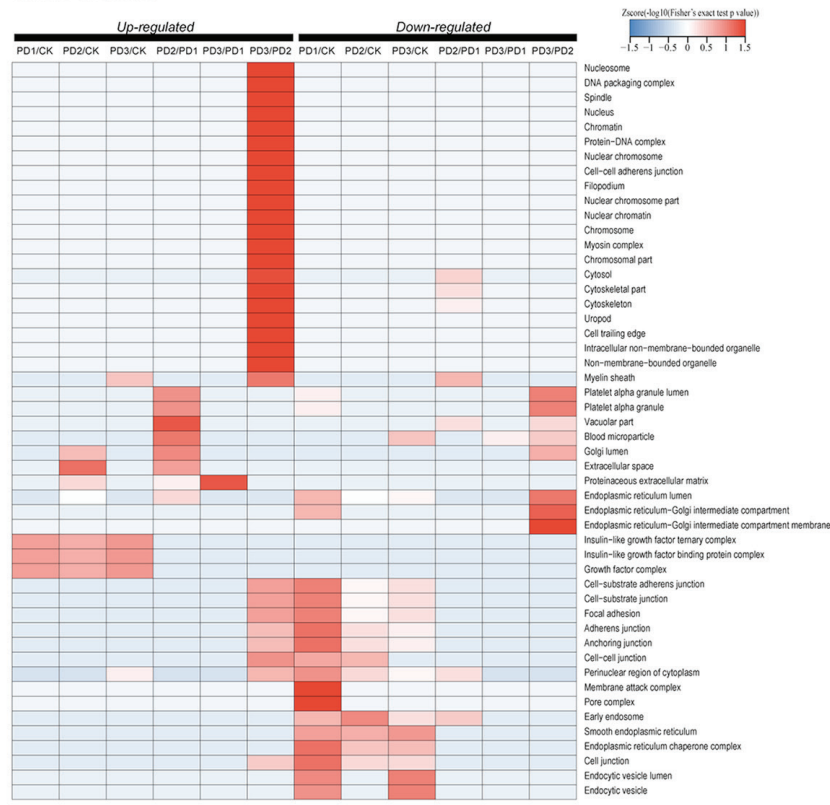

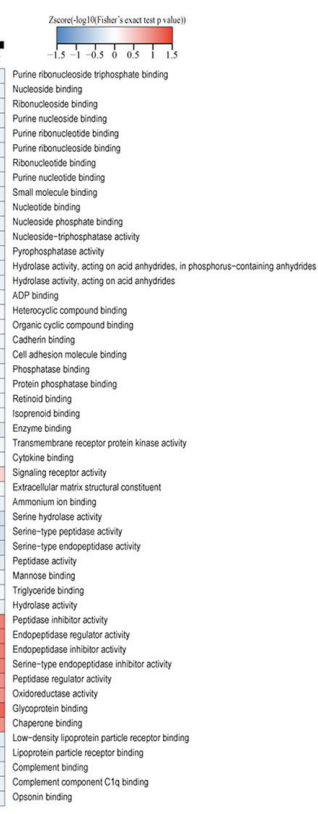

Figure 5. Cluster analysis based on Gene Ontology enrichment analysis. (A) Biological process, (B) cellular component and (C) molecular function. Groups: PD1, PD patients without surgery; PD2, PD patients who had undergone DBS surgery without electrode stimulation; PD3, PD patients who had undergone DBS surgery with 1 month of electrode stimulation; CK, control group without PD. DBS, deep brain stimulation; PD, Parkinson's disease; ER, endoplasmic reticulum.

annotated to the ubiquitin protein ligase activity and transition metal ion binding modules in the present study. In 2010, the homologous protein TRIM9 was identified to be repressed in the brain tissue of patients with $\mathrm{PD}$ and dementia with Lewy bodies (25). Furthermore, TRIM3-associated functions, including ubiquitin ligase activity, were confirmed to be associated with nitrosative stress, which is a critical factor in sporadic PD (26). Similarly, transition metal ions may influence the conformation of amyloidogenic peptides and further affect amyloid formation, which significantly affects the pathogenesis of neurodegenerative diseases (27). Therefore, it may be inferred that CCDC154 and TRIM3 are key proteins in the molecular mechanism of PD by participating in intracellular vesicle, ubiquitin protein ligase and transition metal ion-binding activities.

DBS, commonly known as 'brain pacemaker surgery', is the preferred surgical technique for the treatment of PD, dystonia, essential tremor and other movement-associated neurological disorders in the field of neurosurgery (28). The procedure involves the use of directional technology to implant electrodes into specific nerve nuclei in the brain in order to control the electrical activity of the neurons through high-frequency electrical stimulation, thereby controlling, improving or treating certain clinical diseases (29). In the present study, DBS also affected the expression of proteins. After the surgery, DHH expression was downregulated, whereas NRP2 expression was 
A

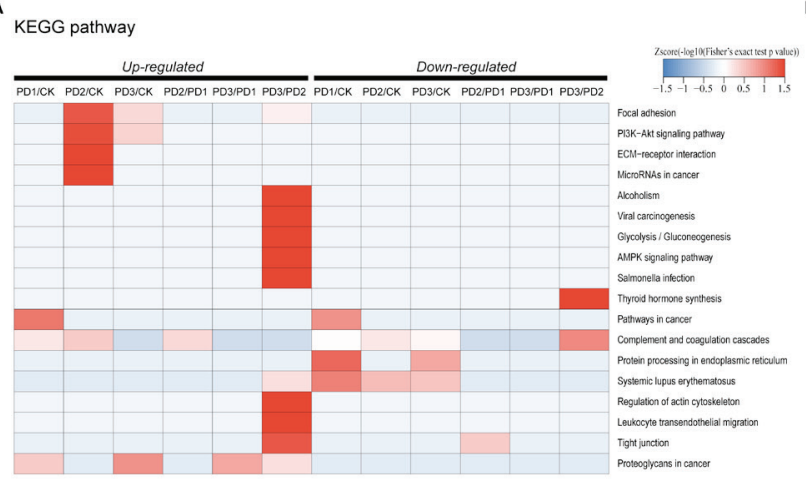

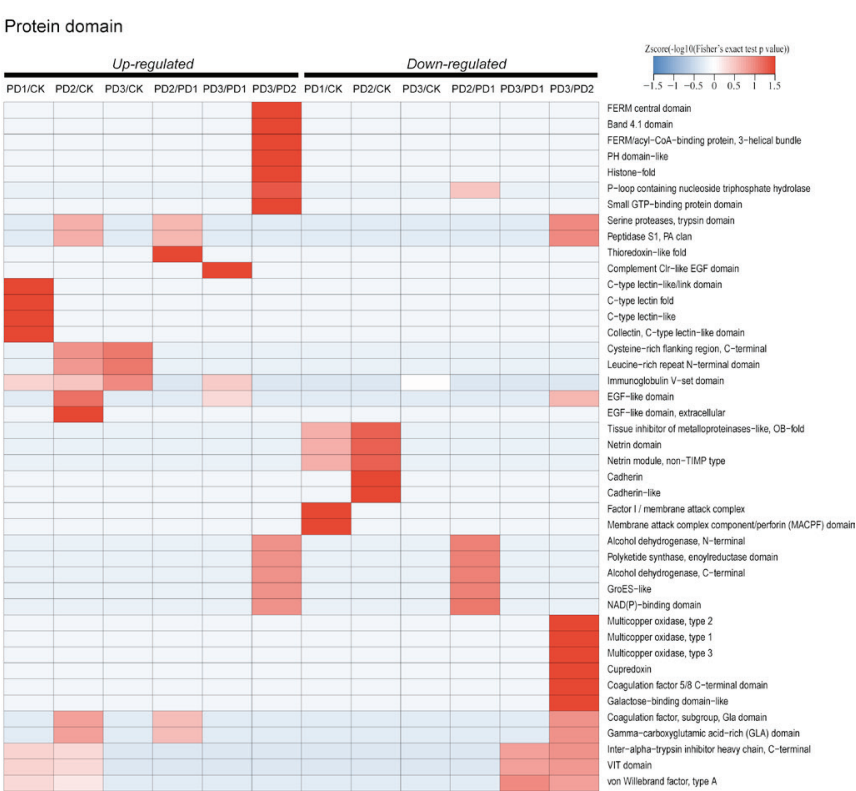

Figure 6. Cluster analysis based on (A) KEGG and (B) protein domain enrichment analyses. Groups: PD1, PD patients without surgery; PD2, PD patients who had undergone DBS surgery without electrode stimulation; PD3, PD patients who had undergone DBS surgery with 1 month of electrode stimulation; CK, control group without PD. DBS, deep brain stimulation; PD, Parkinson's disease; ECM, extracellular matrix; KEGG, Kyoto Encyclopedia of Genes and Genomes; AMPK, Adenosine 5'-monophosphate (AMP)-activated protein kinase; GTP, Guanosine Triphosphate; EGF, Epidermal Growth Factor.

upregulated, participating in the ensheathment of neurons and semaphorin receptor complex, respectively. Dong et al (30) performed an ab initio reconstruction of the transcriptome of mouse cortex tissues, whereupon they detected numerous long non-coding RNAs and determined that upregulated genes and proteins were also involved in the ensheathment of neurons. Furthermore, NRP2 was identified to affect the migration of gonadotropin-releasing hormone-expressing neurons in mice (31). In the Chinese Han population, haplotypes of semaphorin $5 \mathrm{~A}$ were also confirmed to be associated with the risk of PD. Of note, the expression level of CLIC1 was increased after 1 month of electrode stimulation. In response to redox regulation by reactive oxygen species (ROS) derived from NADPH oxidase, the transfer of CLIC1 from the cytoplasmic solute to the plasma membrane was identified to provide a feed-forward mechanism that promoted the NAPDH oxidase production of persistent microglial ROS (32). Above all, DBS surgery may improve the status of patients at the genetic level.

On the basis of these select proteins, PD may be detected at an early stage to achieve early prevention of the progression of disease. In addition, corresponding therapeutic drugs for these proteins may be designed in such a way that the drugs enter the body to specifically select and combine with the lesion site. This would allow for the specific targeting and killing of the diseased cells without affecting the normal cells. Accurate individualized treatment may also be facilitated.

Of note, the present study has certain limitations. Due to the limitation of experimental capacity, lack of confirmation using an animal model of PD was a potential limitation of the present study. Furthermore, the screened biomarkers have not been confirmed by clinical studies. As described by previous studies, cerebrospinal fluid levels of 1B-light chain 3, Beclin-1, lysosome-associated membrane glycoprotein 2 , total tau, phosphorylated tau and alpha-synuclein may be indicators of different types of dementia (33). Whether these screened biomarkers are linked to the above indicators also requires to be confirmed. In future studies by our group, an animal model of PD will be constructed and the expression of the genes screened above will be verified. More importantly, the association of these screened biomarkers with clinical indicators in the PD model and patients will be investigated.

In conclusion, proteomics and bioinformatics approaches and LC-MS methods were combined to facilitate the discovery of novel biomarkers of PD. By detecting significant proteins (e.g., CCDC154, TRIM3, DHH, NRP2 and CLIC1) with high specificity and sensitivity, earlier diagnosis and treatment of $\mathrm{PD}$ may be achieved.

\section{Acknowledgements}

Not applicable.

\section{Funding}

The present study was supported by the Nanjing Medical Science and Technology Development Fund (grant no. ZKX15032) and Jiangsu Provincial Key Research and Development Program (grant no. BE2016614).

\section{Availability of data and materials}

The datasets used and/or analyzed during the present study are available from the corresponding author on reasonable request.

\section{Authors' contributions}

All authors participated in the study design and the manuscript preparation. LZ and WZ made substantial contributions to conception and design. WD and CQ collected the samples and performed the experiments. DG, XJ, WaL and WeL analyzed 
the data. $\mathrm{LZ}$ and $\mathrm{WZ}$ were involved in drafting the manuscript and revising it critically for important intellectual content. All authors read and approved the final manuscript.

\section{Ethics approval and consent to participate}

The use of human tissues was approved by the ethics committee of Nanjing Brain Hospital (approval no. 2016-KY009). All patients or their family members provided written informed consent for participation in the study.

\section{Patient consent for publication}

Not applicable.

\section{Competing interests}

The authors declare that they have no competing interests.

\section{References}

1. Ma XW and Guo RY: Dose-dependent effect of Curcuma longa for the treatment of Parkinson's disease. Exp Ther Med 13: 1799-1805, 2017.

2. Kuroda K, Tatara K, Takatorige T and Shinsho F: Effect of physical exercise on mortality in patients with Parkinson's disease. Acta Neurol Scand 86: 55-59, 1992.

3. Fan JY, Chang BL and Wu YR: Relationships among depression, anxiety, sleep, and quality of life in patients with Parkinson's disease in Taiwan. Parkinsons Dis 2016: 4040185, 2016.

4. Warner TT and Schapira AH: Genetic and environmental factors in the cause of Parkinson's disease. Ann Neurol 53 (Suppl 3): S16-S25, 2003

5. Cummings JL and Mendez MF: Alzheimer's disease: Cognitive and behavioral pharmacotherapy. Conn Med 61: 543-552, 1997.

6. Spitzer AL and Hakim EW: Parkinson's disease: The interplay of medical and surgical treatment options with physical therapy. Acute Care Perspectives 17: 8, 2008.

7. Olanow CW: The pathogenesis of cell death in Parkinson's disease-2007. Mov Disord 22 (Suppl 17): S335-S342, 2007.

8. Blandini F and Armentero MT: Animal models of Parkinson's disease. FEBS J 279: 1156-1166, 2012.

9. Pienaar IS, Daniels WM and Götz J: Neuroproteomics as a promising tool in Parkinson's disease research. J Neural Transm (Vienna) 115: 1413-1430, 2008.

10. Kim SI, Voshol H, van Oostrum J, Hastings TG, Cascio M and Glucksman MJ: Neuroproteomics: Expression profiling of the brain's proteomes in health and disease. Neurochem Res 29 1317-1331, 2004.

11. Monti C, Colugnat I, Lopiano L, Chiò A and Alberio T: Network analysis identifies disease-specific pathways for parkinson's disease. Mol Neurobiol 55: 370-381, 2018.

12. Kiesmann M,Chanson JB, Godet J, Vogel T, Schweiger L, Chayer S and Kaltenbach G: The movement disorders society criteria for the diagnosis of Parkinson's disease dementia: Their usefulness and limitations in elderly patients. J Neurol 260: 2569-2579, 2013.

13. Tyanova S, Temu T and Cox J: The MaxQuant computational platform for mass spectrometry-based shotgun proteomics. Nat Protoc 11: 2301-2319, 2016

14. Okuda S, Yamada T, Hamajima M, Itoh M, Katayama T, Bork P, Goto $S$ and Kanehisa M: KEGG Atlas mapping for global analysis of metabolic pathways. Nucl Acids Res 36 (Web Server Issue): W423-W426, 2008.

15. Horton P, Park KJ, Obayashi T and Nakai K: Protein subcellular localization prediction with WoLF PSORT. Asian Pacific Bioinformatics Conference: 39-48, 2006.

16. Meincke U and Kosinski CM: Treatment of mental disorders in patients with Parkinson's disease. Fortschr Neurol Psychiatr 78: 279-287, 2010 (In German).
17. Peters M, Fitzpatrick R, Doll H, Playford D and Jenkinson C: Does self-reported well-being of patients with Parkinson's disease influence caregiver strain and quality of life? Parkinsonism Relat Disord 17: 348-352, 2011.

18. Schapira AH and Jenner P: Etiology and pathogenesis of Parkinson's disease. Mov Disord 26: 1049-1055, 2011

19. Xu H, Wang Y, Song N, Wang J, Jiang H and Xie J: New progress on the role of glia in iron metabolism and iron-induced degeneration of dopamine neurons in Parkinson's disease. Front Mol Neurosci 10: 455, 2018.

20. Isacson $\mathrm{O}$ and Chung CY: Rab3b for the treatment and prevention of Parkinson's disease. US, 2011.

21. Liao W, Zhao R, Lu L, Zhang R, Zou J, Xu T, Wu C, Tang J, Deng Y and Lu X: Overexpression of a novel osteopetrosis-related gene CCDC154 suppresses cell proliferation by inducing G2/M arrest. Cell Cycle 11: 3270-3279, 2012.

22. Volles MJ and Lansbury PT Jr: Vesicle permeabilization by protofibrillar alpha-synuclein is sensitive to Parkinson's disease-linked mutations and occurs by a pore-like mechanism. Biochemistry 41: 4595-4602, 2002.

23. Islam MS, Nolte H, Jacob W, Ziegler AB, Pütz S, Grosjean Y, Szczepanowska K, Trifunovic A, Braun T, Heumann H, et al: Human R1441C LRRK2 regulates the synaptic vesicle proteome and phosphoproteome in a Drosophila model of Parkinson's disease. Hum Mol Genet 25, 5365-5382 2016.

24. Volz TJ, Farnsworth SJ, King JL, Riddle EL, Hanson GR and Fleckenstein AE: Methylphenidate administration alters vesicular monoamine transporter-2 function in cytoplasmic and membraneassociated vesicles. J Pharmacol Exp Ther 323: 738-745, 2007.

25. Tanji K, Kamitani T, Mori F, Kakita A, Takahashi H and Wakabayashi K: TRIM9, a novel brain-specific E3 ubiquitin ligase, is repressed in the brain of Parkinson's disease and dementia with Lewy bodies. Neurobiol Dis 38: 210-218, 2010.

26. Yao D, Gu Z, Nakamura T, Shi ZQ, Ma Y, Gaston B, Palmer LA, Rockenstein EM, Zhang Z, Masliah E, et al: Nitrosative stress linked to sporadic Parkinson's disease: S-nitrosylation of parkin regulates its E3 ubiquitin ligase activity. Proc Natl Acad Sci USA 101: 10810-10814, 2004.

27. Hoernke M, Koksch B and Brezesinski G: Influence of the hydrophobic interface and transition metal ions on the conformation of amyloidogenic model peptides. Biophys Chem 150: 64-72, 2010.

28. Medical Advisory Secretariat: Deep brain stimulation for Parkinson's disease and other movement disorders: An evidence-based analysis. Ont Health Technol Assess Ser 5: 1-56, 2005.

29. Escudero M, de la Cruz RR and Delgado-García JM: A physiological study of vestibular and prepositus hypoglossi neurones projecting to the abducens nucleus in the alert cat. J Physiol 458: 539-560, 1992.

30. Dong X, Chen K, Cuevas-Diaz DR, You Y, Sloan SA, Zhang Y, Zong S, Cao Q, Barres BA and Wu JQ: Comprehensive identification of long non-coding RNAs in purified cell types from the brain reveals functional LncRNA in OPC fate determination. PLoS Genetics 11: e1005669, 2015.

31. Cariboni A, Davidson K, Rakic S, Maggi R, Parnavelas JG and Ruhrberg C: Defective gonadotropin-releasing hormone neuron migration in mice lacking SEMA3A signalling through NRP1 and NRP2: Implications for the aetiology of hypogonadotropic hypogonadism. Hum Mol Genet 20: 336-344, 2011.

32. Milton RH, Abeti R, Averaimo S, DeBiasi S, Vitellaro L, Jiang L, Curmi PM, Breit SN, Duchen MR and Mazzanti M: CLIC1 function is required for beta-amyloid-induced generation of reactive oxygen species by microglia. J Neurosci 28: 11488-11499, 2008.

33. Hall S, Ohrfelt A, Constantinescu R, Andreasson U, Surova Y, Bostrom F, Nilsson C, Håkan W, Decraemer H, Någga K, et al: Accuracy of a panel of 5 cerebrospinal fluid biomarkers in the differential diagnosis of patients with dementia and/or parkinsonian disorders. Arch Neurol 69: 1445-1452, 2012.

This work is licensed under a Creative Commons Attribution-NonCommercial-NoDerivatives 4.0 International (CC BY-NC-ND 4.0) License. 